\title{
YAP1 Is a Driver of Myofibroblast Differentiation in Normal and Diseased Fibroblasts
}

\author{
Bram Piersma, ${ }^{*}$ Saskia de Rond,${ }^{*}$ Paul M.N. Werker, ${ }^{\dagger}$ Stellar Boo, ${ }^{\ddagger}$ Boris Hinz, ${ }^{\ddagger}$ Marike M. van Beuge, ${ }^{*}$ and Ruud A. Bank*
}

\begin{abstract}
From the Department of Pathology and Medical Biology,* University of Groningen, University Medical Center Groningen, Matrix Research Group, Groningen, The Netherlands; the Department of Plastic Surgery, ${ }^{\dagger}$ University of Groningen, University Medical Center Groningen, Groningen, The Netherlands; and the Laboratory of Tissue Repair and Regeneration, ${ }^{\ddagger}$ Matrix Dynamics Group, Faculty of Dentistry, University of Toronto, Toronto, Ontario, Canada
\end{abstract}

Accepted for publication August 14, 2015.

Address correspondence to Ruud A. Bank, Ph.D., Hanzeplein 1, 9713 GZ, Groningen, The Netherlands. E-mail: r.a.bank@umcg.nl.

\begin{abstract}
Dupuytren disease is a fibrotic disorder characterized by contraction of myofibroblast-rich cords and nodules in the hands. The Hippo member Yes-associated protein 1 (YAP1) is activated by tissue stiffness and the profibrotic transforming growth factor- $\beta 1$, but its role in cell fibrogenesis is yet unclear. We hypothesized that YAP1 regulates the differentiation of dermal fibroblasts into highly contractile myofibroblasts and that YAP1 governs the maintenance of a myofibroblast phenotype in primary Dupuytren cells. Knockdown of YAP1 in transforming growth factor- $\beta 1$-stimulated dermal fibroblasts decreased the formation of contractile smooth muscle $\alpha$-actin stress fibers and the deposition of collagen type I, which are hallmark features of myofibroblasts. Translating our findings to a clinically relevant model, we found that YAP1 deficiency in Dupuytren disease myofibroblasts resulted in decreased expression of ACTA2, COL1A1, and CCN2 mRNA, but this did not result in decreased protein levels. YAP1-deficient Dupuytren myofibroblasts showed decreased contraction of a collagen hydrogel. Finally, we showed that YAP1 levels and nuclear localization were elevated in affected Dupuytren disease tissue compared with matched control tissue and partly co-localized with smooth muscle $\alpha$-actin-positive cells. In conclusion, our data show that YAP1 is a regulator of myofibroblast differentiation and contributes to the maintenance of a synthetic and contractile phenotype, in both transforming growth factor- $\beta 1$-induced myofibroblast differentiation and primary Dupuytren myofibroblasts. (Am J Pathol 2015, 185: 3326-3337; http://dx.doi.org/10.1016/j.ajpath.2015.08.011)
\end{abstract}

Dupuytren disease is a benign fibroproliferative disorder of the hands and fingers of unknown cause, characterized by the formation of myofibroblast-rich cords and nodules. ${ }^{1,2}$ Myofibroblasts are key cells in the pathophysiology of Dupuytren disease and a variety of other fibrotic disorders. ${ }^{1-4}$ Myofibroblasts synthesize excessive amounts of extracellular matrix (ECM) proteins such as collagens, glycoproteins, and proteoglycans. $^{4-6}$ Furthermore, myofibroblasts are characterized by the expression of high levels of smooth muscle (SM) $\alpha$-actin, which enhances their ability to contract tissue. ${ }^{7,8}$ Myofibroblast contraction in Dupuytren disease results in severe contracture of the palmar fascia, with subsequent progressive flexion of the fingers and a substantial reduction in the quality of life. ${ }^{1,2}$ The most potent factor that activates myofibroblasts in fibrotic lesions is transforming growth factor (TGF)- $\beta 1 .^{8-10}$ TGF- $\beta 1$ was shown to be up-regulated in affected Dupuytren tissue, ${ }^{10,11}$ but, up to now, no feasible treatments to inhibit profibrotic signaling in this disease have been found. ${ }^{2}$

TGF- $\beta 1$ was shown to activate the transcriptional coactivator Yes-associated protein 1 (YAP1), which binds to and promotes nuclear accumulation/activity of Smad1 and Smad3. ${ }^{12,13}$ As part of the Hippo signaling cascade, YAP1 was shown to govern organ size by influencing cell proliferation and cell death in both Drosophila and mammals. ${ }^{14,15}$ YAP1 activity is negatively regulated by the Hippo core kinase

\footnotetext{
Supported by the Netherlands Institute for Regenerative Medicine (NIRM) grant FES0908 (R.A.B.), Canadian Institutes of Health Research (CIHR) grants 210820 and 286920 (B.H.), and Collaborative Health Research Program (CIHR/NSERC) grants 1004005 and 413783 (B.H.) UMCG Imaging and Microscopy Center (UMIC) was supported by Netherlands Organisation for Scientific Research (NWO) grant 40-0050698-9021.
}

Disclosures: None declared. 
complex, and inactive Hippo signaling results in YAP1 activation and translocation from the cytosol to the nucleus. In the nucleus, active YAP1 associates with transcription factors and enhances their activity in regulating the expression of genes such as connective tissue growth factor and $\beta 2$ integrin. ${ }^{16}$ Recent studies that used epithelial cells, mesenchymal stem cells, and cancer-associated fibroblasts showed that YAP1 activity and nuclear localization are also regulated by mechanical cues from the ECM such as increased tissue stiffness. ${ }^{17-21}$

Both high tissue stiffness and TGF- $\beta 1$ are prerequisites for myofibroblast differentiation, ${ }^{22}$ which led us to propose a role for YAP1 in regulating fibrogenesis. It is currently not completely known whether and how YAP1 plays a role in myofibroblast differentiation in the context of TGF- $\beta 1$ and the pathophysiology of fibrotic disorders such as Dupuytren disease. Our results show that YAP1 is an important factor in TGF- $\beta 1$-stimulated human dermal fibroblast-to-myofibroblast differentiation in vitro and maintenance of a myofibroblast phenotype in diseased primary Dupuytren myofibroblasts.

\section{Materials and Methods}

\section{Primary Dupuytren Tissue Collection}

Dupuytren disease nodules were obtained from patients undergoing primary limited fasciectomy or dermofasciectomy, at the University Medical Center Groningen. Unaffected transverse ligaments of the palmar aponeurosis from the same patients were used as control tissue. Tissues were either snapfrozen at $-80^{\circ} \mathrm{C}$ for future immunohistochemistry or directly used for the isolation of primary Dupuytren myofibroblasts. Informed written consent was obtained through approval of the local ethics committee (METc 2007/067), in accordance with the Declaration of Helsinki.

\section{Cell Culture}

Dupuytren nodules were minced with a sterile scalpel and incubated with $1 \mathrm{mg} / \mathrm{mL}$ collagenase and $0.1 \mathrm{mg} / \mathrm{mL}$ DNase in Dulbecco's modified Eagle medium (Lonza, Basel, Switzerland) for 2 hours at $37^{\circ} \mathrm{C}$. Digested tissue fragments were filtered through a $70-\mu \mathrm{m}$ cell strainer, and the cell suspension was centrifuged at $300 \times g$ for 10 minutes at $4^{\circ} \mathrm{C}$. Pellets were resuspended in Dulbecco's modified Eagle medium, containing $2 \mathrm{mmol} / \mathrm{L}$ L-glutamine (Lonza), $1 \%$ penicillin/streptomycin (Life Technologies, Carlsbad, CA), supplemented with $10 \%$ fetal bovine serum (Thermo Scientific, Waltham, MA), and seeded in tissue culture polystyrene (TCPS) culture ware. Subcultures were maintained until reaching $80 \%$ to $90 \%$ confluence, before starting experiments. Cells from all donors were used up to passage 4 .

Human dermal fibroblasts were purchased from ATCC (CCD-1093Sk; Wesel, Germany), and subcultures were maintained in Eagle's minimal essential medium (Lonza) that contained $2 \mathrm{mmol} / \mathrm{L}$ L-glutamine and $1 \%$ penicillin/ streptomycin (complete growth medium) and supplemented with $10 \%$ fetal bovine serum.

\section{Transfections and Treatments}

For YAP1 localization experiments, 10,000 cells $/ \mathrm{cm}^{2}$ were seeded on fibronectin-coated polyacrylamide gels with a substrate elasticity of $4 \mathrm{kPa}$. For transfection and stimulation experiments, 15,000 cells $/ \mathrm{cm}^{2}$ were seeded on tissue culture polystyrene culture ware. Cells were transfected with endoribonuclease-prepared siRNA (esiRNA) against YAP1 (62.5 ng/mL; Sigma-Aldrich, St. Louis, MO), and esiRNA against a nonsense sequence $(62.5 \mathrm{ng} / \mathrm{mL}$; Sigma-Aldrich) was used as control. All transfections were performed with Lipofectamine RNAiMax (Life Technologies) in antibioticsfree complete growth medium, according to the manufacturer's instructions. For all cell types, medium was refreshed 8 hours after transfection, and cells were starved for 16 hours in complete growth medium supplemented with $0.5 \%$ fetal bovine serum and $0.17 \mathrm{mmol} / \mathrm{L}$ vitamin $\mathrm{C}$ (L-ascorbic acid 2-phosphate sesquimagnesium hydrate; Sigma-Aldrich). For the generation of myofibroblasts, dermal fibroblasts were stimulated with $10 \mathrm{ng} / \mathrm{mL}$ TGF- $\beta 1$ (PeproTech EC Ltd, London, United Kingdom) in starvation medium for 3 or 7 days. For cell contraction assays, cells were stimulated with TGF- $\beta 1$ in starvation medium for 3 days. Subsequently, 5000 cells $/ \mathrm{cm}^{2}$ were seeded on fibronectin-coated wrinkling silicone substrates, produced as described previously. ${ }^{9}$ Cells were allowed to wrinkle the substrate for 48 hours before analysis. Per experiment, we analyzed 12 high-power fields and counted the total cell numbers and the numbers of wrinkled cells with the use of ImageJ version 1.49a $(\mathrm{NIH}$, Bethesda, MD). Primary Dupuytren myofibroblasts revert to a quiescent state after several passages in vitro. ${ }^{23}$ To generate an activating, profibrotic environment, Dupuytren disease myofibroblasts were therefore stimulated with $10 \mathrm{ng} / \mathrm{mL}$ TGF- $\beta 1$ (PeproTech) in starvation medium for 3 or 7 days. All cells were cultured at $37^{\circ} \mathrm{C}$ in a humidified atmosphere with $5 \% \mathrm{CO}_{2}$.

\section{Collagen Contraction Assay}

Three days after transfection, activated dermal fibroblasts and Dupuytren disease myofibroblasts were seeded in collagen gels with a final concentration of $2.4 \mathrm{mg} / \mathrm{mL}$ rat tail collagen type I (BD, San Jose, CA), $5.8 \mathrm{mmol} / \mathrm{L} \mathrm{NaOH}, 1 \times$ phosphate-buffered saline (PBS), $20 \mathrm{mmol} / \mathrm{L}$ HEPES, 50\% Eagle's minimal essential medium complete growth medium, and 5\% fetal bovine serum. Cells were seeded at a concentration of $2 \times 10^{5} / \mathrm{mL}$ gel. Cells were allowed to build up tension within the gels 3 days before detachment. At time point $\mathrm{t}=0$ minutes, gels were cut loose from the well and allowed to contract. Well plates were scanned at multiple time points on a flatbed scanner. Gel contraction was analyzed with ImageJ. 


\section{RNA Extraction and Real-Time PCR}

For gene expression analysis, total RNA was isolated with the Tissue Total RNA mini kit (Favorgen Biotech Corp., Taiwan), and RNA quantity and purity were determined with UV spectrophotometry (NanoDrop Technologies, Wilmington, DE). One microgram of RNA was reverse transcribed with the RevertAid First Strand cDNA Synthesis Kit (Thermo Scientific). Real-time PCR was performed with SYBR green PCR master mix (Roche, Basel, Switzerland) and a VIIA7 thermal cycling system (Applied Biosystems, Carlsbad, CA). The thermal cycling conditions were $2 \mathrm{mi}-$ nutes at $95^{\circ} \mathrm{C}$ (enzyme activation), followed by 15 seconds at $95^{\circ} \mathrm{C}, 30$ seconds at $60^{\circ} \mathrm{C}$, and 30 seconds at $72^{\circ} \mathrm{C}(40$ cycles). Melting curve analysis was performed to verify the absence of primer dimers. Used primer sequences (forward and reverse, respectively) were as follows: ACTA2, $5^{\prime}$-CTGTTCCAGCCATCCTTCAT- $3^{\prime}$ and $5^{\prime}$-TCATGATGCTGTTGTAGGTGGT-3'; COL1A1， 5'-GCCTCAAGGTATTGCTGGAC- $3^{\prime}$ and $5^{\prime}$-ACCTTGTTTGCCAGGTTCAC-3'; CCN2, 5'-AGCTGACCTGGAAGAGAACATT-3' and $5^{\prime}$-GCTCGGTATGTCTTCATGCTG-3'; YAP1, 5'-AATCCCACTCCCGACAGG- $3^{\prime}$ and $5^{\prime}$-GACTACTCCAGTGGGGGTCA-3'; YHWAZ, 5'-GATCCCCAATGCTTCACAAG- $3^{\prime}$ and $5^{\prime}$-TGCTTGTTGTGACTGATCGAC- $3^{\prime}$. mRNA expression levels of genes from the collagen biosynthesis and remodeling pathway were analyzed with a custommade microfluidic card-based low-density array (Applied Biosystems) and a VIIA7 thermal cycling system, as described previously. ${ }^{24}$ For each gene, fluorescent intensity was related to the fluorescent intensity of the reference gene tyrosine 3-monooxygenase/tryptophan 5-monooxygenase activation protein, $\zeta$ polypeptide, which proved to be most stable out of a selection of eight reference genes. Primers were designed and optimized to have a calculated $95 \%$ to $105 \%$ reaction efficiency.

\section{Immunofluorescence Microscopy}

For immunofluorescence of YAP1, vinculin, and F-actin, cells were washed twice with PBS and fixed with $2 \%$ paraformaldehyde (Sigma-Aldrich) for 10 minutes. For immunofluorescence of SM $\alpha$-actin and collagen type I, cells were washed twice in PBS and fixed with ice-cold methanol/acetone (1:1) for 10 minutes. Fixed cells were permeabilized with $0.5 \%$ Triton X-100 in PBS for 10 minutes. Primary mouse antihuman SM $\alpha$-actin (dilution 1:100; M0851; Dako, Glostrup, Denmark), mouse anti-human collagen type I (dilution 1:1000; ab90395; Abcam, Cambridge, United Kingdom), mouse antihuman vinculin (dilution 1:1000; V9131; Sigma-Aldrich), and rabbit anti-human YAP1 (dilution 1:100; sc-15407; Santa Cruz Biotechnology, Dallas, TX) were diluted in PBS that contained $2.2 \%$ bovine serum albumin (Sanquin reagents, Amsterdam, The Netherlands). Biotinylated goat anti-rabbit IgG (dilution 1:100; E0432; Dako), biotinylated goat anti-mouse IgG2a (dilution 1:100; 1081-08; Southern Biotech, Birmingham, AL), and streptavidin-cyanine-3 (dilution 1:400; Southern Biotech) were used for fluorescent signals. Tetramethylrhodaminelabeled phalloidin (dilution 1:5000; P1951; Sigma-Aldrich) was used to label cytoskeletal F-actin. Nuclei were visualized with DAPI (dilution 1:5000). All wash steps were performed in PBS that contained $0.05 \%$ Tween20 (Sigma-Aldrich). Microphotographs were acquired in a random blind fashion (B.P.) with the use of a Leica DMRA microscope (Leica Microsystems, Rijswijk, The Netherlands), a Tissuefaxs microscopy system (TissueGnostics GmbH, Vienna, Austria), or a Leica TCS SP8 confocal system (Leica Microsystems).

\section{Immunoblotting}

Cells were lyzed with RIPA buffer (Thermo Scientific) supplemented with $1 \%$ protease inhibitor cocktail (Sigma-Aldrich) and $1 \%$ phosphatase inhibitor cocktail (Sigma-Aldrich), and lysates were disrupted with sonication. Protein concentrations were determined with a DC protein assay (Bio-Rad, Hercules, CA), and equal amounts of protein per lane were subjected to SDS gel electrophoresis. Protein transfer to a nitrocellulose membrane was performed with the semidry Transblot Turbo system (Bio-Rad). For dot blot analysis, per sample $1.5 \mu \mathrm{g}$ of protein was spotted on a nitrocellulose membrane. Membranes were dried for 30 minutes and subsequently used for immunodetection. Membranes were blocked in Tris buffered saline (TBS) $+0.5 \%$ Tween20 (Sigma-Aldrich) that contained 5\% milk powder. Primary mouse anti-human SM $\alpha$-actin (dilution 1:500; M0851; Dako), rabbit anti-human $\beta$-actin (dilution 1:1000; ab8227; Abcam), rabbit anti-human collagen type I (dilution 1:1000; ab93095; Abcam), rabbit anti-human glyceraldehyde 3-phosphate dehydrogenase (dilution 1:1000; ab9482; Abcam), rabbit anti-human YAP1 (dilution 1:250; sc15407; Santa Cruz Biotechnology), rabbit anti-human tyrosine 3-monooxygenase/tryptophan 5-monooxygenase activation protein, $\zeta$ polypeptide (dilution 1:1000; ab51129; Abcam) were diluted in TBS-Tween 20 that contained $5 \%$ milk powder. All washing steps were performed in TBS-Tween20. Protein bands were visualized with chemiluminescence and a ChemiDoc imaging system (Bio-Rad). Image analysis was performed with ImageJ (NIH).

\section{Immunohistochemistry on Dupuytren Disease Tissue}

Cryosections $(5 \mu \mathrm{m})$ were dried under a fan and subsequently fixed in acetone for 10 minutes at $-20^{\circ} \mathrm{C}$. Sections were blocked in 10\% goat serum (First Link Ltd, Wolverhampton, United Kingdom) and subsequently incubated with primary rabbit anti-human YAP1 (dilution 1:100; GTX-129151; Genetex, Irvine, CA), and mouse anti-human SM $\alpha$-actin (dilution 1:100; M0851; Dako) in TBS that contained 2.2\% bovine serum albumin. Secondary goat anti-rabbit IgG HRP (dilution 1:100; P0448; Dako) and biotinylated goat antimouse IgG2a (dilution 1:100; 1081-08; Southern Biotech) were diluted in TBS that contained 2\% human serum. Alkaline phosphatase-labeled streptavidin (dilution 1:500; 7100-04; 


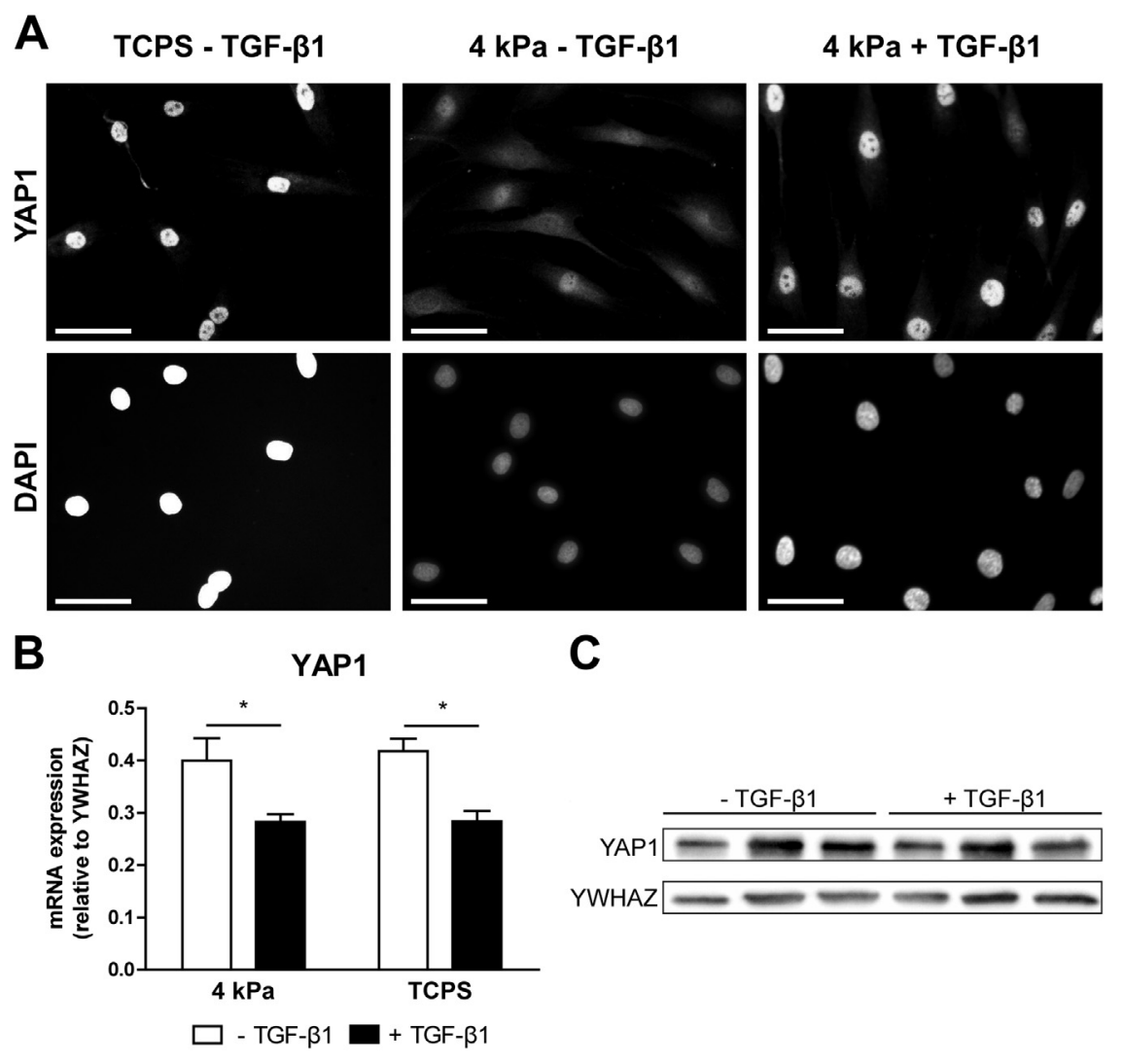

Figure 1 YAP1 activation during TGF- $\beta 1-$ induced myofibroblast differentiation of dermal fibroblasts. A: Representative images of immunofluorescence staining of YAP1 and nuclei (DAPI) on soft polyacrylamide substrates $(4 \mathrm{kPa})$ and tissue culture polystyrene (TCPS) with or without TGF- $\beta 1$ stimulation for 24 hours. B: YAP1 mRNA expression after TGF- $\beta 1$ stimulation on $4 \mathrm{kPa}$ polyacrylamide substrates and TCPS for 72 hours. C: Immunoblot for YAP1 and YWHAZ in dermal fibroblasts cultured on TCPS after 72 hours of stimulation with TGF- $\beta 1$. $n=3$. Two-way analysis of variance with Bonferroni correction: ${ }^{*} P<0.05$. Scale bar $=50 \mu \mathrm{m}$. Original magnification, $\times 400$. TCPS, tissue culture polystyrene; TGF, transforming growth factor; YAP1, Yes-associated protein 1; YWHAZ, tyrosine 3-monooxygenase/tryptophan 5-monooxygenase activation protein, $\zeta$ polypeptide.

Southern Biotech) was diluted in TBS. 3,3'-Diaminobenzidine (ImmPACT DAB; Vector Labs, Burlingham, CA) and Vector Red (Vector Labs) substrates were used for chromogenic development. Nuclei were counterstained with Mayer's hematoxylin (Sigma-Aldrich). Microphotographs were acquired and analyzed with a NuanceFX spectral imaging system (Caliper LS, Hopkinton, MA).

\section{Confocal Analysis of Dupuytren Disease Tissue}

Cryosections $(5 \mu \mathrm{m})$ were dried and fixed in $4 \%$ formaldehyde in PBS for 10 minutes, blocked in 5\% goat and 5\% donkey serum (First Link Ltd), and subsequently incubated with primary rabbit anti-human YAP1 (dilution 1:100; GTX-129151), and mouse anti-human SM $\alpha$-actin (dilution 1:100; M0851; Dako) diluted in PBS that contains 2.2\% bovine serum albumin. Secondary biotinylated goat antirabbit IgG (dilution 1:100; E0432; Dako) and donkey antimouse IgG Alexa Fluor 647 (dilution 1:100; A31571; Life Technologies) were diluted in PBS that contained 2\% human serum. Cyanine-3-labeled streptavidin (dilution 1:500; Southern Biotech) was diluted in PBS + DAPI (dilution 1:5000). Autofluorescence was blocked with a 1-mg/mL solution of Sudan Black B (199664; SigmaAldrich) in $70 \%$ ethanol. Fluorescent images and z-stacks were obtained with a Leica TCS SP8 confocal system (Leica Microsystems), and image files were analyzed and processed with Fiji software version $1.49 \mathrm{v}(\mathrm{NIH}){ }^{25}$

\section{Statistical Analysis}

All data were analyzed with GraphPad Prism version 5.03 (GraphPad Software, La Jolla, CA).

\section{Results}

\section{TGF- $\beta 1$ Activates YAP1 in Dermal Fibroblasts}

To determine whether YAP1 is involved in the differentiation of myofibroblasts, we first cultured human dermal fibroblasts on TCPS in the presence and absence of TGF- $\beta 1$. We found that in unstimulated cells cultured on standard TCPS substrates, YAP1 was already activated as seen by a predominantly nuclear localization (Figure 1A) with no substantial change after TGF- $\beta 1$ treatment (data not shown). To verify if TGF$\beta 1$ could activate YAP1 in dermal fibroblasts, we cultured human dermal fibroblasts on $4 \mathrm{kPa}$ soft fibronectin-coated hydrogels to create a condition with low YAP1 activity. YAP1 localized weakly in both cytoplasm and nucleus in fibroblasts cultured on soft hydrogels, but on TGF- $\beta 1$ stimulation YAP1 translocated to the nucleus (Figure 1A). On both soft hydrogels and TCPS, YAP1 mRNA levels decreased on TGF- $\beta 1$ stimulation for 24 hours, although YAP1 protein levels remained unchanged (Figure 1, B and C). These findings are consistent with a previous report that found reduced YAP1 mRNA levels on injury, whereas protein levels did not change. ${ }^{26}$ 


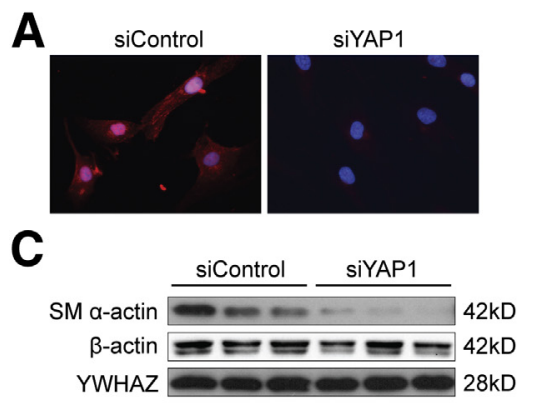

D

E

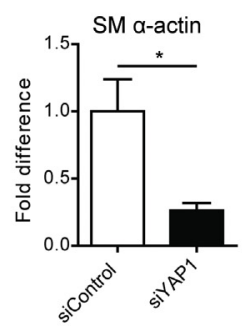

B

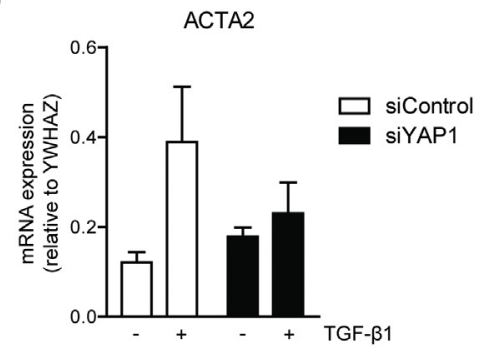

$\mathbf{F}$

G

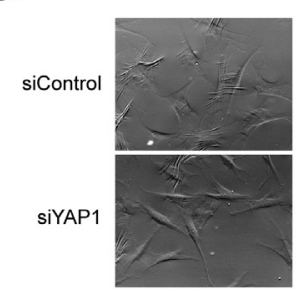

H

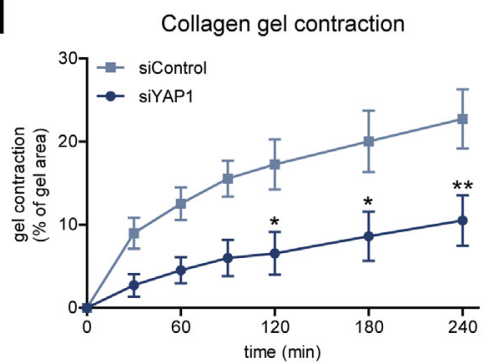

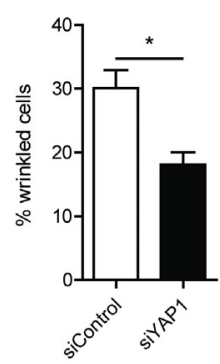

SIYAP1

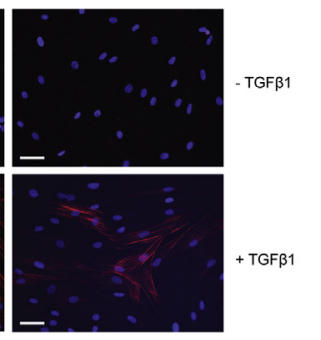

I

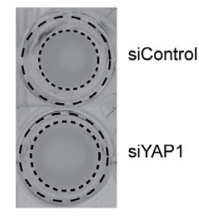

K

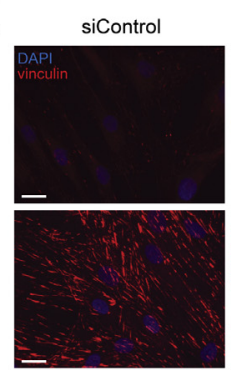

SIYAP1

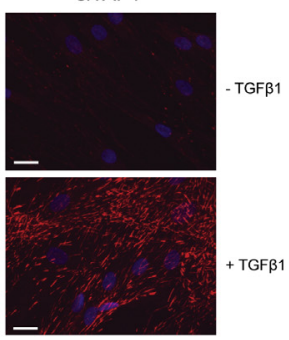

Figure 2 YAP1 knockdown during myofibroblast differentiation cultured on tissue culture polystyrene. A: Representative immunofluorescence photomicrographs of YAP1 staining in dermal fibroblasts treated with nonsense or YAP1 endoribonuclease-prepared siRNA. B: ACTA2 mRNA expression after TGF- $\beta 1$ stimulation for 72 hours. C: Immunoblot for SM $\alpha$-actin, $\beta$-actin, and YWHAZ in dermal fibroblasts after 72 hours of stimulation with TGF- $\beta 1$. D: Quantification of SM $\alpha$-actin immunoblot relative to YWHAZ. E: Representative immunofluorescence photomicrographs of SM $\alpha$-actin (red) and DAPI (blue) staining in dermal fibroblasts after 72 hours. F and G: Wrinkling assay on dermal fibroblasts stimulated with TGF- $\beta 1$ after 48 hours. The percentage of wrinkled cells was calculated over 12 high-power fields per experiment. $\mathbf{H}$ and I: Collagen gel contraction assay. H: Gels were photo scanned every $30 \mathrm{mi}-$ nutes up to 240 minutes. I: Representative image scans of collagen gels at time point $t=240$ minutes. J: Representative immunofluorescence photomicrographs of F-actin (red) and DAPI (blue) staining in dermal fibroblasts. K: Representative photomicrographs of vinculin (red) and DAPI (blue) immunofluorescence staining in dermal fibroblasts. Bonferroni correction was performed on panels $\mathbf{B}$ and $\mathbf{H}$. Data are expressed as means $\pm \operatorname{SEM}(\mathbf{B}, \mathbf{D}, \mathbf{F}$, and $\mathbf{H}) . n=3$ independent experiments $(\mathbf{B}-\mathbf{K})$. Two-way analysis of variance with Bonferroni correction: ${ }^{*} P<0.05,{ }^{*} P<0.01(\mathbf{H})$; paired Student's t-test: ${ }^{*} P<0.05$ (D and F). Scale bars: $100 \mu \mathrm{m}$ (E and $\mathbf{J}) ; 25 \mu \mathrm{m}(\mathbf{K})$. Original magnification: $\times 400(\mathbf{A}) ; \times 200(\mathbf{E}, \mathbf{G}, \mathbf{J}$, and $\mathbf{K})$. ACTA2, actin, $\alpha 2$; SM, smooth muscle; TGF, transforming growth factor; YAP1, Yes-associated protein 1; YWHAZ, tyrosine 3-monooxygenase/tryptophan 5 -monooxygenase activation protein, $\zeta$ polypeptide.

\section{YAP1 Is Required for Myofibroblast Differentiation} in Vitro

We performed esiRNA-mediated knockdown of YAP1 during TGF- $\beta 1$-induced myofibroblast differentiation and verified YAP1 knockdown efficiency by immunofluorescence (Figure 2A). In unstimulated cells, YAP1 knockdown had no effect on the expression of ACTA2, the gene encoding the SM $\alpha$-actin protein. Furthermore, on TGF- $\beta 1$ stimulation, YAP1-deficient cells did not show up-regulation of ACTA2 mRNA, whereas control cells showed a trend toward increased ACTA2 expression (Figure 2B). Interestingly, we did not find any differences in ACTA2 mRNA levels between cells cultured on 4 and 100-kPa polyacrylamide hydrogels and fibroblasts cultured on TCPS (Supplemental Figure S1). Because
YAP1 is constitutively nuclear/active on TCPS, subsequent experiments into the effects of YAP1 deficiency during TGF- $\beta 1$ stimulation were performed on TCPS. In this setting we found that SM $\alpha$-actin protein up-regulation on 72 hours of TGF- $\beta 1$ stimulation was significantly lower after YAP1 knockdown, as shown by immunoblotting (Figure 2, C and D), which was confirmed by immunofluorescence (Figure 2E). We also investigated if $\beta$-actin, another component of the actin cytoskeleton, was affected by YAP1 deficiency, but we did not observe any changes in the expression of $\beta$-actin (Figure 2C). Expression of SM $\alpha$-actin was shown to increase fibroblast contraction. ${ }^{22}$ To test the contraction potential of myofibroblasts after YAP1 knockdown, we performed a single cell wrinkling assay. ${ }^{22}$ Transfected cells were stimulated with TGF$\beta 1$ for 3 days, seeded on fibronectin-coated silicone-based 


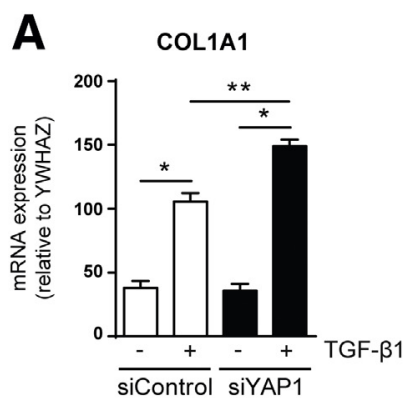

B
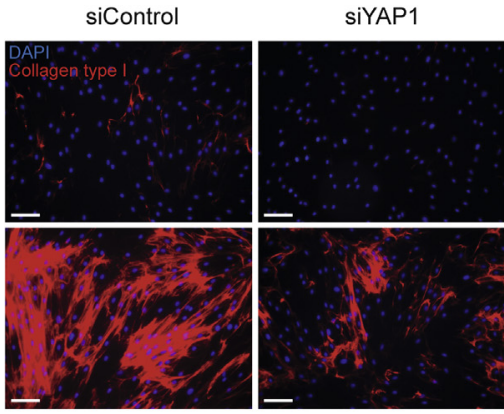

- TGF $\beta 1$

D

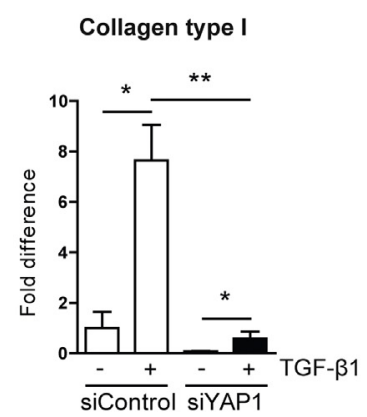

Figure 3 YAP1 knockdown disrupts collagen synthesis in dermal fibroblasts cultured on tissue culture polystyrene. A: COL1A1 mRNA expression after TGF- $\beta 1$ stimulation for 72 hours. B: Representative photomicrographs of collagen type I (red) and DAPI (blue) immunofluorescence staining in dermal fibroblasts after 72 hours. Dot blot for collagen type I in dermal fibroblasts stimulated with TGF- $\beta 1$ for 72 hours; immunoblot for collagen type I (C) and quantification of dot blot analysis (D). Data are represented as means \pm SEM (A and D). $n=3$ independent experiments (A, C, and $\mathbf{D})$. Two-way analysis of variance with Bonferroni correction: ${ }^{*} P<0.05,{ }^{*} P<<0.01$. Scale bar $=100 \mu \mathrm{m}$. Original magnification, $\times 200$. COL1A1, collagen type 1, $\alpha 1$; TGF, transforming growth factor; YAP1, Yes-associated protein 1; YWHAZ, tyrosine 3-monooxygenase/tryptophan 5 -monooxygenase activation protein, $\zeta$ polypeptide. wrinkling substrates, and monitored for 2 days. Knockdown of YAP1 resulted in a decreased percentage of wrinkling/contracting cells (Figure 2, F and G). We further showed that YAP1 has a significant role in the myofibroblast-mediated contraction of three-dimensional collagen gels (Figure 2, H and I). Interestingly, we found no changes in the levels of F-actin and the focal adhesion component vinculin in either unstimulated or stimulated cells (Figure 2, J and $\mathrm{K}$ ).

In addition to being highly contractile cells, myofibroblasts produce high amounts of collagenous ECM that contribute to the progression of fibrotic disorders. Thus, we investigated whether YAP1 is involved in collagen production by myofibroblasts. YAP1-deficient cells showed increased mRNA levels of COL1A1 after stimulation with TGF- $\beta 1$ (Figure 3A). Again, we found no differences between fibroblasts cultured on 4 and $100 \mathrm{kPa}$ polyacrylamide hydrogels and fibroblasts cultured on TCPS (Supplemental Figure S1). As expected, stimulation of control cells with TGF- $\beta 1$ resulted in a marked increase in collagen type I deposition. Interestingly, this increase was attenuated in the absence of YAP1, as seen by immunofluorescence (Figure 3B). These results were confirmed by dot blot analysis, which also showed significantly lower collagen type I levels in YAP1-deficient cells compared with YAP1expressing cells (Figure 3, C and D). We next performed a gene expression analysis of genes involved in the collagen biosynthesis pathway, because an altered expression pattern in these genes could account for the impaired collagen type I deposition in YAP1-deficient cells. We found that, of the 28 genes examined, 4 genes (PLOD1, P4HA2, LOXL3, and $P C O L C E)$ were significantly up-regulated in YAP1-deficient cells, compared with controls, after stimulation with TGF- $\beta 1$ (Table 1). However, up-regulation of these genes suggests an increase in the biosynthesis and remodeling of collagens, whereas the opposite was found for collagen type I. We did not see a down-regulation in any of the examined genes involved in collagen biosynthesis.

\section{YAP1 Is Necessary for Maintenance of Dupuytren Disease Myofibroblasts}

We next examined whether we could translate our findings to a more clinically relevant setting by studying YAP1 in primary human Dupuytren myofibroblasts and tissue. Dupuytren disease is a fibroproliferative disorder of the hands and fingers, characterized by excessive ECM synthesis and contracture of the palmar fascia by myofibroblasts. Dupuytren myofibroblasts quickly revert to a quiescent state when cultured in vitro. ${ }^{23}$ However, at baseline, quiescent Dupuytren myofibroblasts from all donors showed higher levels of SM $\alpha$-actin than unstimulated dermal fibroblasts (Supplemental Figure S2). Because the collagenous tissue in Dupuytren patients has increased stiffness, ${ }^{2}$ we hypothesized that YAP1 activity may be increased in primary Dupuytren myofibroblasts. Interestingly, after 72 hours of culture on $4 \mathrm{kPa}$ soft fibronectin-coated hydrogels YAP1 still localized primarily to the nucleus (Supplemental Figure S3). This suggests that, on compliant substrates, YAP1 remains active in quiescent Dupuytren myofibroblasts. YAP1 knockdown in quiescent Dupuytren myofibroblasts resulted in a decrease of mRNA levels of ACTA2, COL1A1, and CCN2 (Figure 4A). YAP1 knockdown did not change protein levels of SM $\alpha$-actin in inactivated cells (Figure 4, B and C). We then investigated if we could show the effect of YAP1 deficiency in an activating environment by 
Table 1 mRNA Expression Array Collagen Biosynthesis and Remodeling

\begin{tabular}{|c|c|c|c|c|}
\hline Gene name & Gene symbol & $\begin{array}{l}\text { Mean expression } \\
\text { siControl* }^{*}\end{array}$ & $\begin{array}{l}\text { Mean } \\
\text { expression } \\
\text { siYAP1* }\end{array}$ & $\begin{array}{l}\text { Fold } \\
\text { difference }^{\dagger}\end{array}$ \\
\hline Procollagen-lysine 2-oxoglutarate 5-dioxygenase 1 & PLOD1 & 0.3980 & 0.7569 & $1.90^{\ddagger}$ \\
\hline Procollagen-lysine 2-oxoglutarate 5-dioxygenase 2 & PLOD2 & 0.3676 & 0.5191 & 1.41 \\
\hline Procollagen-lysine 2-oxoglutarate 5-dioxygenase 3 & PLOD3 & 0.2477 & 0.4415 & 1.78 \\
\hline Prolyl 4-hydroxylase $\alpha$ polypeptide I & P4HA1 & 1.317 & 2.038 & 1.55 \\
\hline Prolyl 4-hydroxylase $\alpha$ polypeptide II & P4HA2 & 0.0077 & 0.0147 & $1.90^{\ddagger \ddagger}$ \\
\hline Prolyl 4-hydroxylase $\alpha$ polypeptide III & P4HA3 & 0.1253 & 0.2097 & 1.55 \\
\hline Prolyl 4-hydroxylase beta polypeptide & $P 4 H B$ & 2.667 & 3.228 & 1.21 \\
\hline Leucine proline-enriched proteoglycan (leprecan) 1 & LEPRE1 & 0.1986 & 0.2470 & 1.24 \\
\hline Leprecan-like 1 & LEPREL1 & 0.0052 & 0.0123 & 2.36 \\
\hline Leprecan-like 2 & LEPREL2 & 0.0636 & 0.1443 & 2.27 \\
\hline Lysyl oxidase & LOX & 2.888 & 2.857 & 0.99 \\
\hline Lysyl oxidase-like 1 & LOXL1 & 0.3596 & 0.3988 & 1.11 \\
\hline Lysyl oxidase-like 2 & $\angle O X L 2$ & 1.466 & 1.314 & 0.90 \\
\hline Lysyl oxidase-like 3 & $\angle O X L 3$ & 0.0241 & 0.0387 & $1.61^{\ddagger \ddagger \ddagger}$ \\
\hline Lysyl oxidase-like 4 & LOXL4 & 0.0962 & 0.1207 & 1.25 \\
\hline Serpin peptidase inhibitor HSP47 & SERPINH1 & 2.213 & 2.501 & 1.13 \\
\hline ADAM metallopeptidase with thrombospondin type 1 motif 2 & ADAMTS2 & 0.5648 & 0.7577 & 1.34 \\
\hline ADAM metallopeptidase with thrombospondin type 1 motif 3 & ADAMTS3 & 0.0003 & 0.0009 & 2.65 \\
\hline ADAM metallopeptidase with thrombospondin type 1 motif 14 & ADAMTS14 & 0.0035 & 0.0070 & 2.03 \\
\hline Procollagen C-endopeptidase enhancer & PCOLCE & 0.8288 & 1.271 & $1.53^{\ddagger}$ \\
\hline Procollagen C-endopeptidase enhancer 2 & PCOLCE2 & 0.0133 & 0.0217 & 1.63 \\
\hline FK506 binding protein $1065 \mathrm{kDa}$ & FKBP10 & 1.171 & 1.995 & 1.70 \\
\hline Solute carrier family 39 (zinc transporter) member 13 & SLC39A13 & 0.1795 & 0.2419 & 1.35 \\
\hline Matrix metallopeptidase 1 (interstitial collagenase) & MMP1 & 0.0123 & 0.0299 & 2.43 \\
\hline Matrix metallopeptidase 13 (collagenase 3 ) & MMP13 & 0.0004 & 0.0007 & 1.49 \\
\hline Matrix metallopeptidase 14 (membrane-inserted) & MMP14 & 0.4899 & 0.8093 & 1.65 \\
\hline TIMP metallopeptidase inhibitor 1 & TIMP1 & 2.278 & 3.559 & 1.56 \\
\hline Cathepsin $\mathrm{K}$ & CTSK & 0.1503 & 0.2584 & 1.72 \\
\hline
\end{tabular}

mRNA expression of human dermal fibroblasts stimulated with TGF- $\beta 1$ for 3 days.

${ }^{*}$ mRNA expression relative to expression of YWHAZ $(n=3)$.

${ }^{\dagger}$ Fold-difference mRNA expression of siYAP1 compared with siControl. Data were analyzed with a two-way analysis of variance, with Bonferroni correction.

${ }_{\ddagger}^{\ddagger} P<0.05,{ }^{\ddagger \ddagger} P<0.01, \stackrel{\ddagger \ddagger}{ } P<0.001$.

TGF, transforming growth factor; YAP1, Yes-associated protein 1; YWHAZ, tyrosine 3-monooxygenase/tryptophan 5-monooxygenase activation protein, $\zeta$ polypeptide.

stimulation with TGF- $\beta 1$. On TGF- $\beta 1$ stimulation, SM $\alpha$-actin levels were lower in YAP1-deficient cells from four donors, but overall we found no statistical differences compared with control cells (Figure 4, D and E). Furthermore, we found no differences in collagen type 1 levels between YAP1-deficient and control cells stimulated with TGF- $\beta 1$ (Figure 4, F and G). We then tested if Dupuytren myofibroblasts require YAP1 for their contractile phenotype. Indeed, YAP1 deficiency resulted in decreased contraction of three-dimensional collagen gels compared with gels populated with YAP1-expressing cells (Figure 4, $\mathrm{H}$ and $\mathrm{I}$ ).

\section{Dupuytren Myofibroblasts Show Increased YAP1 Expression}

To verify if YAP1 is involved in the pathology of Dupuytren disease, we performed double immunohistochemical and immunofluorescence staining for YAP1 and SM $\alpha$-actin in matched nodule and control tissues obtained from eight patients. Immunohistochemical analysis revealed that both YAP1 and SM $\alpha$-actin were significantly increased in Dupuytren nodular tissue compared with control tissue (Figure 5). As described before, ${ }^{27}$ we found that Dupuytren nodules were highly heterogeneous with multiple SM $\alpha$-actin ${ }^{+}$ areas, surrounded by SM $\alpha$-actin ${ }^{-}$tissue regions (Figure 5). YAP1 was expressed at low levels in control tissues (Figure 5, G-J). Notably, YAP1 was highly expressed throughout the Dupuytren nodules, in both SM $\alpha$-actin ${ }^{+}$and SM $\alpha$-actin tissue regions. Moreover, we found an increased association of YAP1 staining with nuclear staining in affected Dupuytren nodule tissue compared with control tissue (Figure 5). We could confirm nuclear localization of YAP1 in both SM $\alpha$-actin ${ }^{+}$and SM $\alpha$-actin ${ }^{-}$cells in Dupuytren nodule tissue with the use of confocal microscopy (Figure 6), although we also found cells to be negative for both YAP1 and SM $\alpha$-actin. These data are consistent with previous studies that suggest that the cell population in the 
A

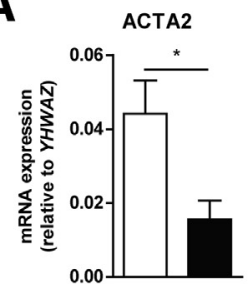

B

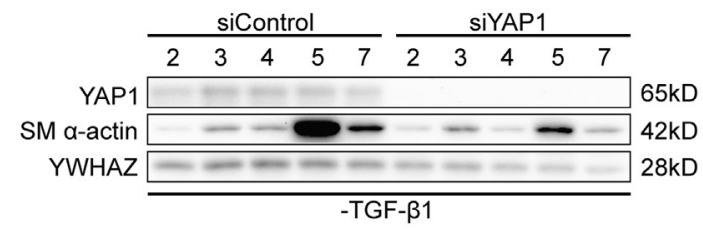

D

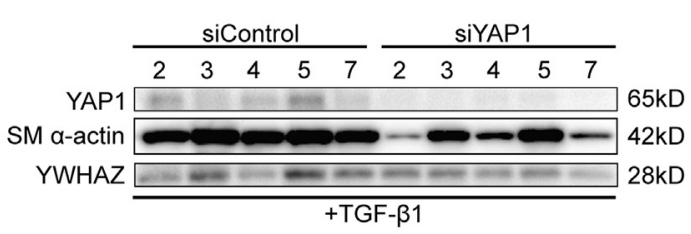

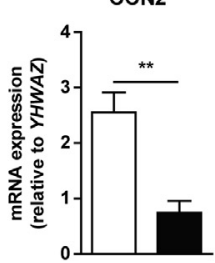

C

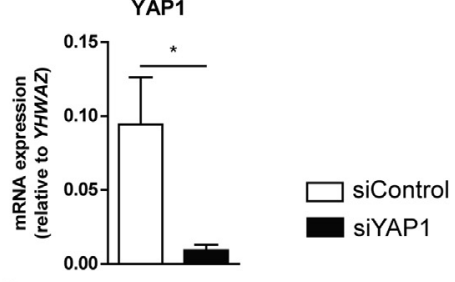

SM $\alpha$-actin

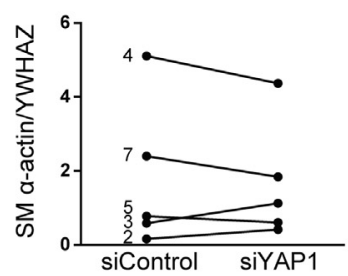

E

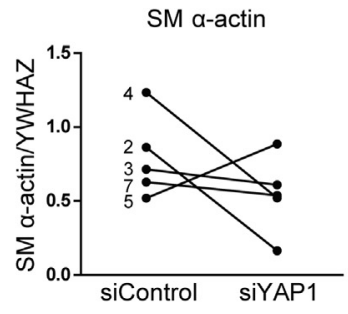

$\mathbf{F}$

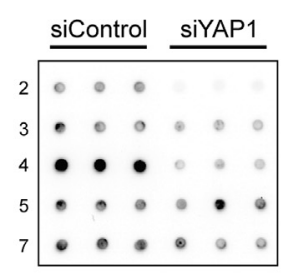

G

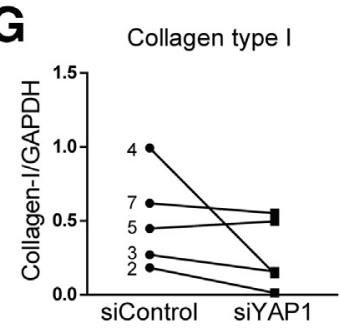

H

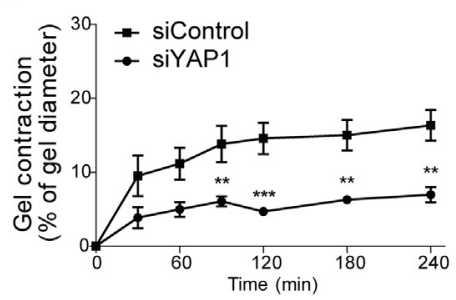

I

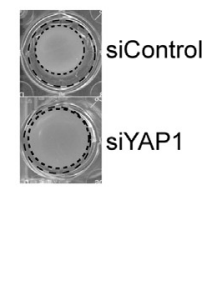

Figure 4 YAP1 knockdown in Dupuytren disease myofibroblasts cultured on tissue culture polystyrene. A: mRNA expression of ACTA2, COL1A1, CCN2, and YAP1 in Dupuytren disease myofibroblasts after culture in starvation medium for 7 days. B and C: Immunoblot analysis of YAP1, SM $\alpha$-actin, and YWHAZ in Dupuytren disease myofibroblasts, cultured in starvation medium for 7 days. C: Quantification of SM $\alpha$-actin immunoblot relative to YWHAZ; lines show paired data for each donor. D and E: Immunoblot analysis of YAP1, SM $\alpha$-actin, and YWHAZ in Dupuytren disease myofibroblasts, stimulated with TGF- $\beta 1$ for 7 days. E: Quantification of SM $\alpha$-actin immunoblot relative to YWHAZ; lines show paired data for each donor. $\mathbf{F}$ and $\mathbf{G}$ : Immunoblot analysis of collagen type I in Dupuytren disease myofibroblasts from donors, stimulated with TGF- $\beta 1$ for 7 days. G: Quantification of collagen type I immunoblot relative to GAPDH; lines show paired data for each donor. $\mathbf{H}$ and I: Dupuytren disease myofibroblast contraction in a three-dimensional collagen gel; gels were photo scanned every 30 minutes up to 240 minutes. I: Representative image scans of collagen gels at time point $t=240$ minutes. The dashed lines indicate the well rim (outer line) and the gel edge (inner line). Wilcoxon matched pairs test was performed for panels $\mathbf{A}, \mathbf{C}, \mathbf{E}$, and $\mathbf{G}$. Data are represented as means \pm SEM (A). $n=4$ donors $\left(\mathbf{A}, \mathbf{H}\right.$, and I) $; n=5$ donors $(\mathbf{B}-\mathbf{G})$. Wilcoxon matched pairs test: ${ }^{*} P<0.05,{ }^{*} P<0.01(\mathbf{A})$; two-way analysis of variance with Bonferroni correction: ${ }^{*} P<<0.01, * * * P<0.001$ (H and I). ACTA2, actin, $\alpha 2$; CCN2, connective tissue growth factor; COL1A1, collagen type 1, $\alpha 1$; GAPDH, glyceraldehyde 3-phosphate dehydrogenase; SM, smooth muscle; TGF, transforming growth factor; YAP1, Yes-associated protein 1; YWHAZ, tyrosine 3-monooxygenase/ tryptophan 5-monooxygenase activation protein, $\zeta$ polypeptide.

Dupuytren lesion is highly heterogeneous. ${ }^{27}$ Moreover, we did not find a correlation between cells with nuclear YAP1 and SM $\alpha$-actin staining.

\section{Discussion}

Studies have identified YAP1 as a link between the Hippo and TGF- $\beta 1$ signaling cascades. ${ }^{12,13}$ Understanding how TGF- $\beta 1$ signaling connects with the Hippo pathway member YAP1 in relation to profibrotic signaling is therefore necessary. Our data provide evidence that YAP1 plays a distinct role in the regulation of myofibroblast differentiation and maintenance. We showed that YAP1 contributes to the establishment of a contractile and synthetic phenotype of fibroblasts activated by TGF- $\beta 1$ and primary Dupuytren myofibroblasts. Finally, the high expression and nuclear localization of YAP1 protein in Dupuytren nodular tissue suggest a role for YAP1 in the pathology of Dupuytren disease.

YAP1 is a transcriptional co-activator, known for its role in mechanobiology in several cell types. ${ }^{18-21}$ In cancer-associated fibroblasts, YAP1 activity is necessary for the up-regulation of 

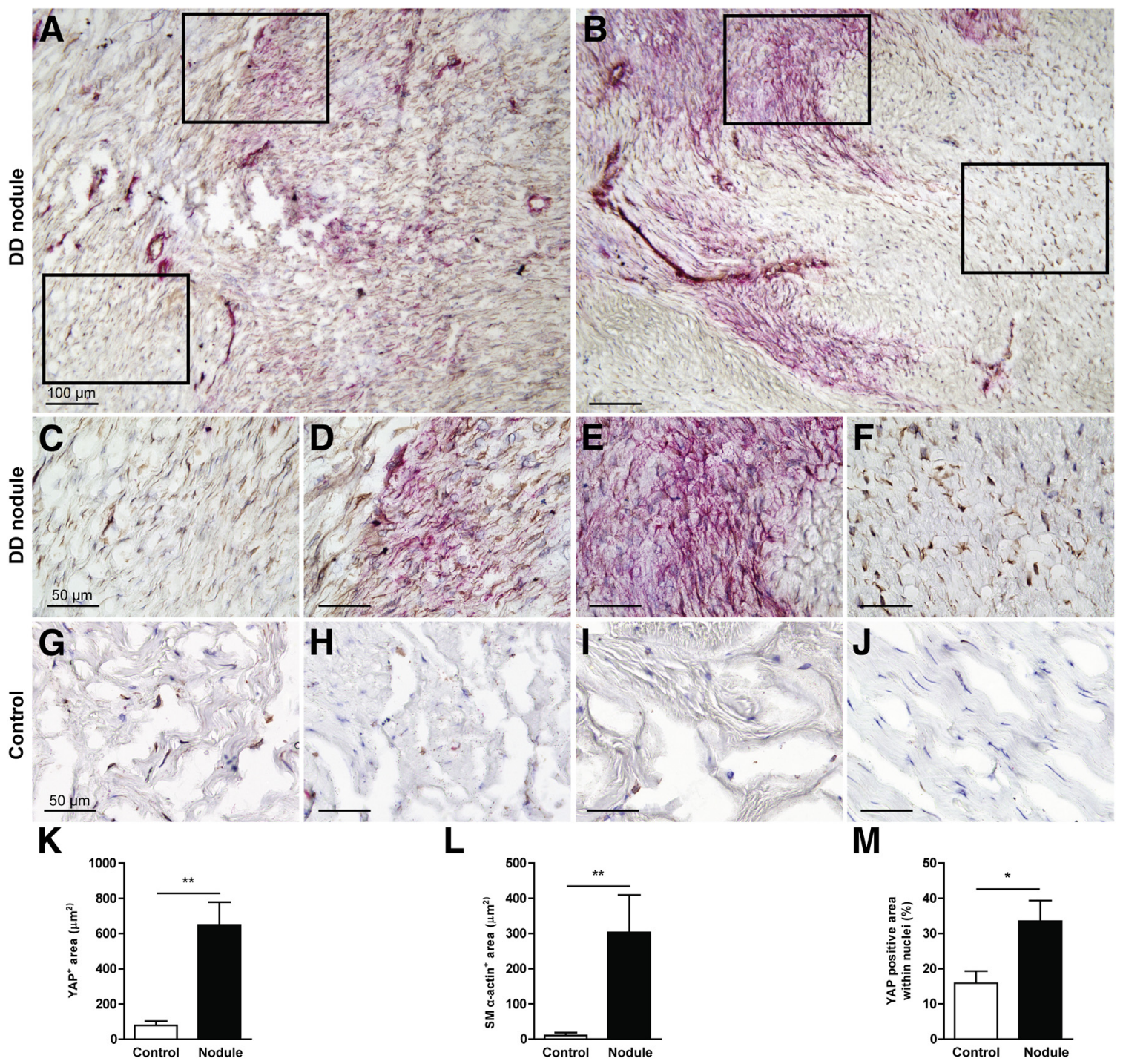
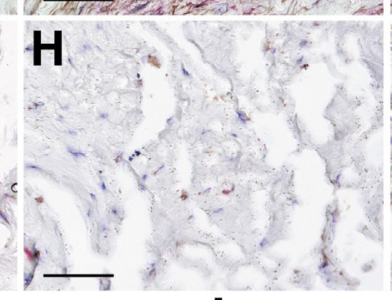

$\mathbf{L}$

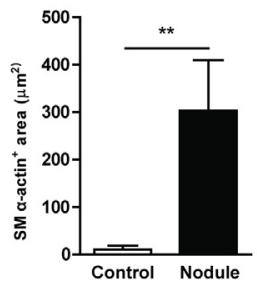

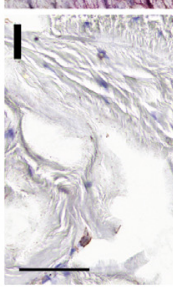

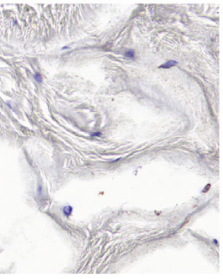

M
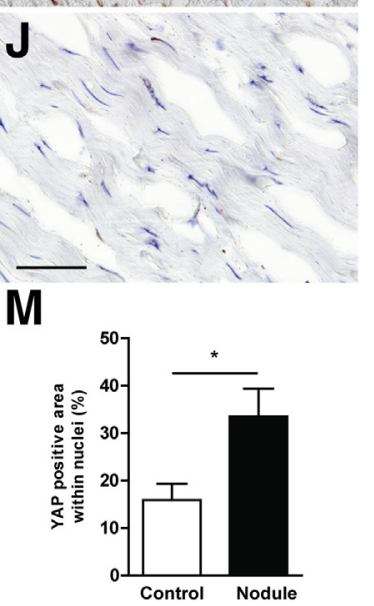

Figure 5 IHC staining for YAP1 and SM $\alpha$-actin in Dupuytren disease nodules and healthy control tissues. A and B: Representative photomicrographs show an overview of IHC staining for YAP1 (brown) and SM $\alpha$-actin (magenta) on Dupuytren disease nodule cryosections. Nuclei are stained with hematoxylin (blue). C-F: High magnification microphotographs of boxed areas from $\mathbf{A}$ and $\mathbf{B}$ show heterogeneity of Dupuytren disease nodular tissue. G-J: High magnification microphotographs of IHC staining for YAP1 and SM $\alpha$-actin on control tissue. K: Quantification of IHC YAP1 staining. L: Quantification of IHC SM $\alpha$-actin staining. M: Quantification of YAP $1^{+}$area within nuclear staining. Data are expressed as means \pm SEM $(\mathbf{K}-\mathbf{M}) . n=8(\mathbf{K}$ and $\mathbf{L}) ; n=7(\mathbf{M})$. Wilcoxon matched pairs test: ${ }^{*} P<0.05,{ }^{* *} P<0.01$. Original magnification: $\times 100(\mathbf{A}$ and $\mathbf{B}) ; \times 400(\mathbf{C}-\mathbf{J})$. DD, Dupuytren disease donor; IHC, immunohistochemical; SM, smooth muscle; YAP1, Yes-associated protein 1.

the cytoskeletal components myosin light chain 2 and myosin heavy chain 10. YAP1 deficiency in these cells resulted in decreased ECM remodeling and contraction. ${ }^{19}$ A recent study on idiopathic pulmonary fibrosis also found both YAP1 and TAZ involved in matrix stiffness-related fibrotic signaling. ${ }^{20}$ Consistently, we show that the contraction of collagen gels by both dermal (myo)fibroblasts and Dupuytren myofibroblasts is influenced by active YAP1. However, it is currently unknown why the YAP1-deficient Dupuytren myofibroblasts showed reduced gel contraction, because we found no differences in SM $\alpha$-actin and collagen type I protein levels compared with control cells.

A possible explanation may lie in altered expression or distribution of other components of the actin cytoskeleton or focal adhesion proteins. Indeed, in cancer-associated fibroblasts and mouse fibroblasts, YAP1 activity was required for the association of paxillin and vinculin with the focal adhesion complex, suggesting that the integrity of focal adhesions is disrupted when YAP1 is absent. However, we found that the distribution of the focal adhesion component vinculin was unchanged in YAP1-deficient dermal fibroblasts, which suggests that the functions of YAP1 may vary, depending on the source of the fibroblasts. The same studies suggested that YAP1-deficient cells have a decreased capacity to deposit collagen, which is consistent with the results found in our study. Although we found that mRNA levels of COL1A1 were increased after YAP1 knockdown, we found a dramatic reduction in collagen deposition in both dermal fibroblasts and Dupuytren 
A

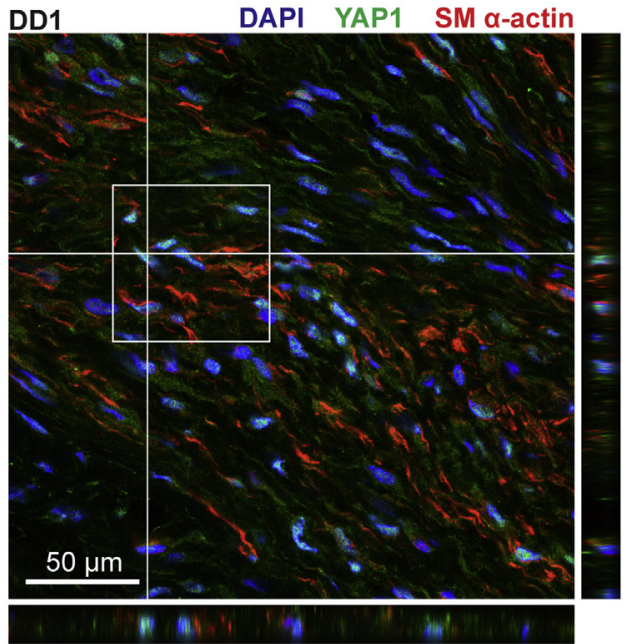

DD1

C

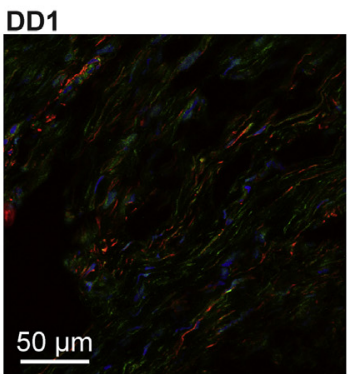

DD5

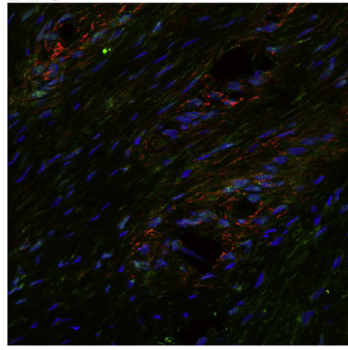

DD2

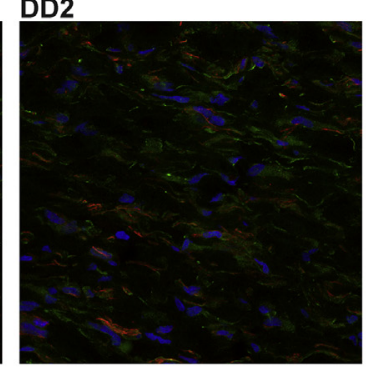

DD6

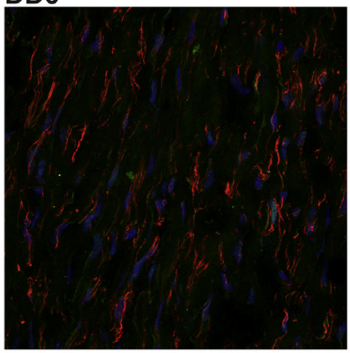

B

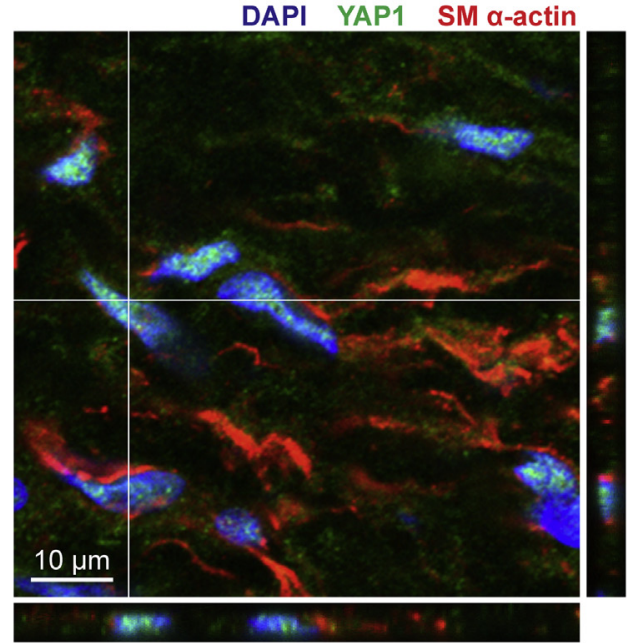

DD3
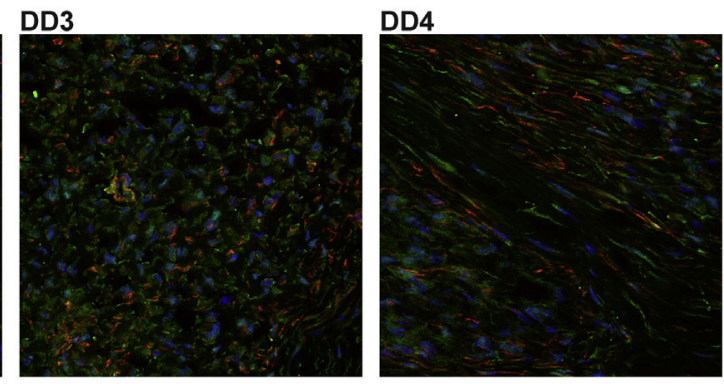

DD7

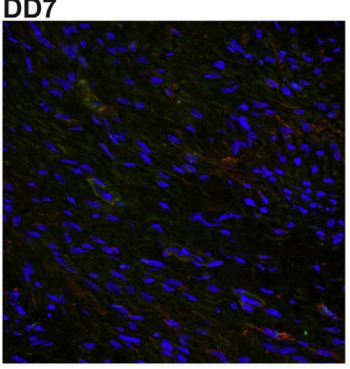

DD8

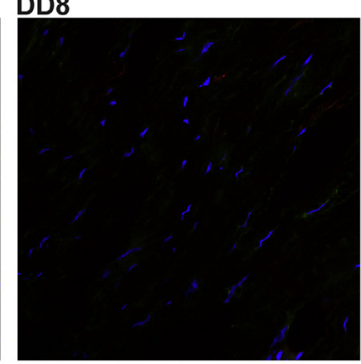

DAPI YAP1 SM $\alpha$-actin

Figure 6 Representative confocal photomicrographs of immunofluorescence-stained Dupuytren nodule tissues. A: Overview of nodule tissue from DD1, showing YAP1, SM $\alpha$-actin, and nuclei (DAPI) staining. Orthogonal views show 40 z-stacks $>10 \mu \mathrm{m}$. B: Detailed view of boxed area in A shows co-localization of YAP1 and nuclei and perinuclear staining of SM $\alpha$-actin. C: Representative confocal photomicrographs of nodule tissues show YAP1, SM $\alpha$-actin, and DAPI staining in all donors. DD1 to DD5 show high levels of YAP1 with both nuclear and cytoplasmic localization, and varying levels of SM $\alpha$-actin. DD6 shows medium levels of YAP1 and high SM $\alpha$-actin levels. DD7 shows high levels of cytoplasmic YAP1 with low nuclear localization and low levels of SM $\alpha$-actin. DD8 shows low levels of both YAP1 and SM $\alpha$-actin. $n=8$ (C). Original magnification: $\times 630$ (A and C). DD, Dupuytren disease donor; SM, smooth muscle; YAP1, Yes-associated protein 1.

myofibroblasts. We hypothesized that the high mRNA expression may represent a rescue mechanism of the cell to compensate for defects in the collagen biosynthesis pathway. This might also be the case for the observed up-regulation of the collagen-modifying enzymes PLOD1, P4HA2, LOXL3, and PCOLCE. In fact, we did not find a down-regulation of genes involved in the collagen biosynthesis pathway that could explain the dramatic reduction in collagen type I deposition. Alternatively, the discrepancy between collagen mRNA expression and protein deposition may lie in altered rate of mRNA translation. Previous work on the correlation between mRNA expression, mRNA stability, and protein translation revealed that the translation rate of collagen type I depends more on translation than transcription. ${ }^{28}$ Finally, YAP1 deficiency increases the expression of Let7 miRNAs, which are known to target collagen transcripts. ${ }^{29,30}$ Future clarification of the collagen biosynthesis pathway in YAP-deficient fibroblasts should shed light on these findings.

TGF- $\beta 1-$ stimulated fibroblasts (myofibroblasts) are well known for their excessive production of ECM components and the ability to remodel and contract the tissue around them. ${ }^{4,5}$ Activation by TGF- $\beta 1$ results in an up-regulation of ECM components such as collagen type I and fibronectin and a maturation of the cytoskeleton as seen by increased 
levels of SM $\alpha$-actin. ${ }^{6}$ Although YAP1 activation in response to TGF- $\beta 1$ was shown, ${ }^{13,19}$ the effect of activation of YAP1 in response to TGF- $\beta 1$ in myofibroblasts remains elusive. Interestingly, we found that YAP1 mRNA levels were lower in TGF- $\beta 1-$ stimulated cells on both soft and stiff substrates, suggesting a decrease in YAP1 signaling. However, regarding the increased nuclear localization of YAP1 on TGF- $\beta 1$ stimulation, lower mRNA levels may serve as a negative feedback mechanism, because YAP1 protein levels did not change. These results are in agreement with data from a dextran sodium sulfate-induced colitis and regeneration model that showed an increase in YAP1 protein, but a decrease in YAP1 mRNA. ${ }^{26}$ It is possible that YAP1 binds to Smad2/3 and promotes the nuclear translocation of the SMAD2/3/4 complex. YAP1 deficiency may thus result in decreased Smad2/3 levels in the nucleus, ${ }^{13}$ impairing TGF- $\beta 1$-induced expression of target genes, such as ACTA2, ${ }^{31}$ as we found in our study. In this regard, YAP1 may function similar to its counterpart TAZ. ${ }^{32}$ In addition, a recent study places SM $\alpha$-actin upstream of YAP, because it found a direct link between SM $\alpha$-actin expression and YAP activity in the differentiation of human mesenchymal stem cells toward myofibroblasts. ${ }^{33}$ Although we did not find a correlation between SM $\alpha$-actin and YAP in Dupuytren nodule tissue, whether a direct link exists in other fibrotic disorders remains unclear. ${ }^{33}$

Furthermore, it remains unclear how active YAP1 promotes the induction of a synthetic and contractile myofibroblast phenotype. One of the possibilities is through the induction of the YAP1 target CCN2, a matricellular protein that is thought to enhance TGF- $\beta 1$ signaling, creating a forward feedback loop. ${ }^{34}$ YAP1 deficiency results in a decreased expression of CCN2, which in turn may result in decreased TGF- $\beta 1$ signaling. ${ }^{34}$ In this manner, active YAP1 could invoke a possible feedback loop in which TGF- $\beta 1$ and $C C N 2$ signaling are increased. ${ }^{34-36}$ In support of this hypothesis, we found a decreased expression of CCN2 in YAP1-deficient Dupuytren disease myofibroblasts, suggesting a role for CCN2 signaling via YAP1 in these cells. Indeed, both TGF- $\beta 1$ and CCN2 signaling are enhanced in Dupuytren disease myofibroblasts and play a role in the pathogenic phenotype of these cells. ${ }^{11,37,38}$

We were able to show that YAP1 functions as a regulator of contractility in Dupuytren disease myofibroblasts. One limitation of this study is that the Dupuytren cells had to be subcultured up to passage 4 on plastic culture ware. These conditions may have altered the initial phenotype of the cells which could have influenced our results. ${ }^{23}$ Nevertheless, the trend seen in decreased protein levels of SM $\alpha$-actin and collagen type I suggest that similar mechanisms apply to dermal fibroblasts and Dupuytren myofibroblasts. Moreover, high expression of YAP1 in both SM $\alpha$-actin ${ }^{+}$and SM $\alpha$-actin ${ }^{-}$cells in affected tissue suggests a prominent role in affected Dupuytren disease tissue. Increased association of YAP1 staining with nuclear staining suggests that YAP1 activity in affected Dupuytren nodule tissue is increased compared with control tissue. We could confirm the nuclear localization of
YAP1 with confocal analysis but also found $\mathrm{YAP}^{-}$cells and cells that showed primarily cytoplasmic localization of YAP1. Whether the $\mathrm{YAP}^{+} / \mathrm{SM} \alpha$-actin ${ }^{-}$cells are a quiescent or deactivated form of the Dupuytren disease myofibroblasts remains unknown. Although SM $\alpha$-actin is expressed in contractile-differentiated myofibroblasts, collagen-producing proto-myofibroblasts are SM $\alpha$-actin ${ }^{-}$but can remodel the ECM around them, ${ }^{8,39}$ suggesting that the cells within the Dupuytren disease lesion are in a different state of activation. Notably, high YAP1 expression and increased nuclear localization in affected Dupuytren disease tissue may be caused by both TGF- $\beta 1$ signaling and high tissue stiffness because YAP1 is a known mechanotransducer. ${ }^{13,18}$ Future studies should be focused on unraveling the cross talk of YAP1 with TGF- $\beta 1$ signaling in Dupuytren disease and, more generally, fibrotic disorders.

We have found that YAP1 plays an evident role in myofibroblast differentiation and maintenance in both TGF$\beta 1-$ stimulated dermal fibroblasts and primary Dupuytren disease myofibroblasts and that Dupuytren disease nodule tissue is characterized by elevated YAP1 expression. Our results agree with and augment previous studies that identified YAP1 as key molecule in fibrogenesis. ${ }^{19,20,33}$ YAP1 is emerging as a protein acting on the crossroads of multiple signal transduction pathways such as TGF- $\beta 1$ and CCN2 signaling. Altogether, our data suggest that YAP1 acts as a major regulator of myofibroblasts in fibrosis.

\section{Acknowledgments}

We thank Miriam Boersema for the discussions during the experimental setup and data analysis and Anna Wil Bosma for technical assistance.

B.P., M.M.v.B., and R.A.B. conceived the experimental setup; B.P., S.d.R., and M.M.v.B. performed all experiments; S.B. generated the fibronectin-coated silicon substrates and suggested the experimental setup; P.M.N.W. coordinated all the surgical procedures; B.P. analyzed all data and drafted the manuscript; B.P., M.M.v.B., B.H., and R.A.B. wrote and revised the manuscript. All authors had final approval of the submitted and approved manuscript.

\section{Supplemental Data}

Supplemental material for this article can be found at http://dx.doi.org/10.1016/j.ajpath.2015.08.011.

\section{References}

1. Ross JA, Annan JH: Dupuytren's contracture; a clinical review. Ann Surg 1952, 134:186-194

2. Shih B, Bayat A: Scientific understanding and clinical management of Dupuytren disease. Nat Rev Rheumatol 2010, 6:715-726

3. Tomasek J, Rayan GM: Correlation of alpha-smooth muscle actin expression and contraction in Dupuytren's disease fibroblasts. J Hand Surg Am 1995, 20:450-455 
4. Hinz B, Phan SH, Thannickal VJ, Prunotto M, Desmouliere A, Varga J, De Wever O, Mareel M, Gabbiani G: Recent developments in myofibroblast biology: paradigms for connective tissue remodeling. Am J Pathol 2012, 180:1340-1355

5. Wynn TA: Cellular and molecular mechanisms of fibrosis. J Pathol 2008, 214:199-210

6. Klingberg F, Hinz B, White ES: The myofibroblast matrix: implications for tissue repair and fibrosis. J Pathol 2013, 229:298-309

7. Gabbiani G, Ryan GB, Majno G: Presence of modified fibroblasts in granulation tissue and their possible role in wound contraction. Experientia 1971, 27:549-550

8. Tomasek JJ, Gabbiani G, Hinz B, Chapponier C, Brown RA: Myofibroblasts and mechano-regulation of connective tissue remodelling. Nat Rev Mol Cell Biol 2002, 3:349-363

9. Klingberg F, Chow ML, Koehler A, Boo S, Buscemi L, Quinn TM, Costell M, Alman BA, Genot E, Hinz B: Prestress in the extracellular matrix sensitizes latent TGF- $\beta 1$ for activation. J Cell Biol 2014, 207: 283-297

10. Krause $\mathrm{C}$, Kloen $\mathrm{P}$, Ten Dijke P: Elevated transforming growth factor $\beta$ and mitogen-activated protein kinase pathways mediate fibrotic traits of Dupuytren's disease fibroblasts. Fibrogenesis Tissue Repair 2011, 4:14

11. Badalamente MA, Sampson SP, Hurst LC, Dowd A, Miyasaka K: The role of transforming growth factor beta in Dupuytren's disease. J Hand Surg Am 1996, 21:210-215

12. Alarcón C, Zaromytidou AI, Xi Q, Gao S, Yu J, Fujisawa S, Barlas A, Miller AN, Manova-Todorova K, Macias MJ, Sapkota G, Pan D, Massagué J: Nuclear CDKs drive Smad transcriptional activation and turnover in BMP and TGF-beta pathways. Cell 2009, 139:757-769

13. Varelas X, Samavarchi-Tehrani P, Narimatsu M, Weiss A, Cockburn K, Larsen BG, Rossant J, Wrana JL: The Crumbs complex couples cell density sensing to Hippo-dependent control of the TGF- $\beta$ SMAD pathway. Dev Cell 2010, 19:831-844

14. Basu S, Totty NF, Irwin MS, Sudol M, Downward J: Akt phosphorylates the Yes-associated protein, YAP, to induce interaction with 14-3-3 and attenuation of p73-mediated apoptosis. Mol Cell 2003, 11:11-23

15. Camargo FD, Gokhale S, Johnnidis JB, Fu D, Bell GW, Jaenisch R, Brummelkamp TR: YAP1 increases organ size and expands undifferentiated progenitor cells. Curr Biol 2007, 17:2054-2060

16. Zhao B, Ye X, Yu J, Li L, Li W, Li S, Yu J, Lin JD, Wang CY, Chinnaiyan AM, Lai ZC, Guan KL: TEAD mediates YAPdependent gene induction and growth control. Genes Dev 2008, 22:1962-1971

17. Fujii M, Nakanishi H, Toyoda T, Tanaka I, Kondo Y, Osada H, Sekido Y: Convergent signaling in the regulation of connective tissue growth factor in malignant mesothelioma: TGF $\beta$ signaling and defects in the Hippo signaling cascade. Cell Cycle 2012, 11:3373-3379

18. Dupont S, Morsut L, Aragona M, Enzo E, Giulitti S, Cordenonsi M, Zanconato F, Le Digabel J, Forcato M, Bicciato S, Elvassore N, Piccolo S: Role of YAP/TAZ in mechanotransduction. Nature 2011, 474:179-183

19. Calvo F, Ege N, Grande-Garcia A, Hooper S, Jenkins RP, Chaudhry SI, Harrington K, Williamson P, Moeendarbary E, Charras G, Sahai E: Mechanotransduction and YAP-dependent matrix remodelling is required for the generation and maintenance of cancerassociated fibroblasts. Nat Cell Biol 2013, 15:637-646

20. Liu F, Lagares D, Choi KM, Stopfer L, Marinković A, Vrbanac V, Probst CK, Hiemer SE, Sisson TH, Horowitz JC, Rosas IO, Fredenburgh LE, Feghali-Bostwick C, Varelas X, Tager AM, Tschumperlin DJ: Mechanosignaling through YAP and TAZ drives fibroblast activation and fibrosis. Am J Physiol Lung Cell Mol Physiol 2015, 308:L344-L357

21. Aragona M, Panciera T, Manfrin A, Giulitti S, Michielin F, Elvassore N, Dupont S, Piccolo S: A mechanical checkpoint controls multicellular growth through YAP/TAZ regulation by actin-processing factors. Cell 2013, 154:1047-1059
22. Hinz B, Celetta G, Tomasek JJ, Gabbiani G, Chaponnier C: Alphasmooth muscle actin expression upregulates fibroblast contractile activity. Mol Biol Cell 2001, 12:2730-2741

23. Rehman S, Xu Y, Dunn WB, Day PJ, Westerhoff HV, Goodacre R, Bayat A: Dupuytren's disease metabolite analyses reveals alterations following initial short-term fibroblast culturing. Mol BioSyst 2012, 8: 2274-2288

24. Hosper NA, Bank RA, van den Berg PP: Human amniotic fluidderived mesenchymal cells from fetuses with a neural tube defect do not deposit collagen type I protein after TGF- $\beta 1$ stimulation in vitro. Stem Cells Dev 2014, 23:555-562

25. Schindelin J, Arganda-Carreras I, Frise E, Kaynig V, Longair M, Pietzsch T, Preibisch S, Rueden C, Saalfeld S, Schmid B, Tinevez JY, White DJ, Hartenstein V, Eliceiri K, Tomancak P, Cardona A: Fiji: an open-source platform for biological-image analysis. Nat Methods 2012, 9:676-682

26. Cai J, Zhang N, Zheng Y, de Wilde RF, Maitra A, Pan D: The Hippo signaling pathway restricts the oncogenic potential of an intestinal regeneration program. Genes Dev 2010, 24:2383-2388

27. Verjee LS, Midwood K, Davidson D, Essex D, Sandison A, Nanchahal J: Myofibroblast distribution in Dupuytren's cords: correlation with digital contracture. J Hand Surg Am 2009, 34A:1785-1794

28. Schwanhaüsser B, Busse D, Li N, Dittmar G, Schuchhardt J, Wolf J, Chen W, Selbach M: Global quantification of mammalian gene expression control. Nature 2011, 473:337-342

29. Chaulk SG, Lattanzi VJ, Hiemer SE, Fahlman RP, Varelas X: The Hippo pathway effectors TAZ/YAP regulate dicer expression and microRNA biogenesis through Let-7. J Biol Chem 2014, 289: 1886-1891

30. Makino K, Jinnin M, Hirano A, Yamane K, Eto M, Kusano T, Honda N, Kajihara I, Makino T, Sakai K, Masuguchi S, Fukushima S, Ihn H: The downregulation of microRNA let-7a contributes to the excessive expression of type I collagen in systemic and localized scleroderma. J Immunol 2013, 190:3905-3915

31. Hu B, Wu Z, Phan SH: Smad3 mediates transforming growth factorbeta-induced alpha-smooth muscle actin expression. Am J Respir Cell Mol Biol 2003, 29:397-404

32. Varelas X, Sakuma R, Samavarchi-Tehrani P, Peerani R, Rao BM, Dembowy J, Yaffe MB, Zandstra PW, Wrana JL: TAZ controls Smad nucleocytoplasmic shuttling and regulates human embryonic stem-cell self-renewal. Nat Cell Biol 2008, 10:837-848

33. Talele NP, Fradette J, Davies JE, Kapus A, Hinz B: Expression of a-smooth muscle actin determines the fate of mesenchymal stromal cells. Stem Cell Reports 2015, 4:1016-1030

34. Abreu JG, Ketpura NI, Reversade B, De Robertis EM: Connectivetissue growth factor (CTGF) modulates cell signalling by BMP and TGF-beta. Nat Cell Biol 2002, 4:599-604

35. Leask A, Holmes A, Black CM, Abraham DJ: Connective tissue growth factor gene regulation. Requirements for its induction by transforming growth factor-beta 2 in fibroblasts. J Biol Chem 2003, 278:13008-13015

36. Van Beek JP, Kennedy L, Rockel JS, Bernier SM, Leask A: The induction of CCN2 by TGFbeta1 involves Ets-1. Arthritis Res Ther 2006, 8:R36

37. Satish L, Gallo PH, Baratz ME, Johnson S, Kathju S: Reversal of TGF$\beta 1$ stimulation of $\alpha$-smooth muscle actin and extracellular matrix components by cyclic AMP in Dupuytren's-derived fibroblasts. BMC Musculoskelet Disord 2011, 12:113

38. Igarashi A, Nashiro K, Kikuchi K, Sato S, Ihn H, Fujimoto M, Grotendorst GR, Takehara K: Connective tissue growth factor gene expression in tissue sections from localized scleroderma, keloid, and other fibrotic skin disorders. J Invest Dermatol 1996, 106:729-733

39. Hinz B, Mastrangelo D, Iselin CE, Chapponier C, Gabbiani G: Mechanical tension controls granulation tissue contractile activity and myofibroblast differentiation. Am J Pathol 2001, 159: $1009-1020$ 